\title{
Permutable SOS (symmetry operational similarity)
}

\author{
Sang-Wook Cheong $\mathbb{D}^{1 凶}$, Seongjoon $\mathrm{Lim}^{1}$, Kai $\mathrm{Du}^{1}$ and Fei-Ting Huang ${ }^{1}$
}

Based on symmetry consideration, quasi-one-dimensional (1D) objects, relevant to numerous observables or phenomena, can be classified into eight different types. We provide various examples of each 1D type and discuss their symmetry operational similarity (SOS) relationships, which are often permutable. A number of recent experimental observations, including current-induced magnetization in polar or chiral conductors, non-linear Hall effect in polar conductors, spin-polarization of tunneling current to chiral conductors, and ferro-rotational domain imaging with linear gyration are discussed in terms of (permutable) SOS. In addition, based on (permutable) SOS, we predict a large number of new phenomena in low symmetry materials that can be experimentally verified in the future.

npj Quantum Materials (2021)6:58; https://doi.org/10.1038/s41535-021-00346-1

\section{INTRODUCTION}

The concept of SOS (symmetry operational similarity) ${ }^{1,2}$ between specimen constituents (periodic crystallographic or magnetic lattices in an external magnetic field, electric fields or stress, and also their time evolution) and measuring probes/quantities (observables such as transmitting electrons, phonons, magnons, photons in various polarization states, including electrons or photons with spin or orbital angular momentum, bulk magnetization or polarization, and also experimental setups to measure, e.g., Hall-type effects) in relation to broken symmetries is powerful to understand and predict observable physical phenomena in low symmetry materials ${ }^{3}$. Precisely speaking, this SOS relationship means that specimen constituents have identical or more (but not less) broken symmetries, compared with measuring probes/ quantities. In other words, in order to have an SOS relationship, specimen constituents cannot have higher symmetries than measuring probes/quantities do. The power of the SOS approach lies in providing readily understandable views of otherwise complex and unintuitive phenomena in low-symmetry materials.

In conjunction with the earlier publications, we here discuss (quasi-)one-dimensional objects (simply referred to as 1D objects): objects can be specimen constituents (periodic lattices in the presence of external perturbations) or measuring probes/quantities (observables), and (quasi-)1D refers objects that have a certain rotation symmetry around the 1D direction, and all rotational symmetry, possibly except $C_{2}$ symmetry, is broken around any directions perpendicular to the 1D direction. Note that periodic crystallographic or magnetic lattices can have two, three, four, or sixfold rotational symmetries $\left(C_{2}, C_{3}, C_{4}\right.$, or $C_{6}$ symmetries in the standard crystallographic notations, respectively), 1D specimen constituents must have at least, $C_{2}$ or $C_{3}$ symmetry around the 1D direction, and broken all of $C_{3}, C_{4}$, and $C_{6}$ symmetries around any directions perpendicular to the 1D direction. One example of 1D objects is cycloidal spins having SOS with polarization $(\boldsymbol{P})$, and cycloidal spins do have $C_{2}$ symmetry around $\boldsymbol{P}$, but no rotation symmetry, including $C_{2}$, around any direction perpendicular to the $\boldsymbol{P}$ direction ${ }^{1}$.

Herein, we use these symmetry operation notations: $\mathbf{R}=$ twofold rotation with the rotation axis perpendicular to the 1D object direction, $\mathbf{R}=$ twofold rotation with the rotation axis along the 1D object direction, $\mathbf{I}=$ space inversion, $\mathbf{M}=$ mirror reflection with the mirror perpendicular to the 1D object direction, $\mathbf{M}=$ mirror reflection with the mirror plane containing the $1 \mathrm{D}$ object direction, $\mathbf{T}=$ time reversal. In the standard crystallographic notations, "R, $\mathbf{R}, \mathbf{I}, \mathbf{M}, \mathbf{M}$, and $\mathbf{T}^{\prime}$ are $C_{2 \perp}, C_{2 \|}, \overline{1}, m_{\perp}, m_{\|}, 1^{\prime}$, respectively ${ }^{1}$. Our notations are natural and intuitive to consider 1D objects. Emphasize that since we consider 1D objects, translational as well as rotational symmetry operations along the 1D object direction are freely allowed ${ }^{1}$. For example, $\mathbf{T}$ symmetry in a simple 1D antiferromagnet is considered to be not broken since translation is freely allowed. In addition, if the symmetry is preserved after a symmetry operation followed by a rotation around the 1D object direction, then we say that the symmetry is not broken.

Hlinka has proposed that there exist eight kinds of vectorlike physical quantities in terms of their invariance under spatiotemporal symmetry operations ${ }^{4}$. In fact, our 1D objects are similar to the vectorlike physical quantities in terms of symmetry, so there are eight kinds of 1D objects. We will call them as $\mathscr{D}, \mathscr{C}, \mathscr{P}, \boldsymbol{a}, \mathscr{D}^{\prime}, \mathscr{C}^{\prime}, \mathscr{P}^{\prime}, \boldsymbol{\alpha}^{\prime}$. Here $\mathscr{D}, \mathscr{C}, \mathscr{P}$, and $\boldsymbol{\alpha}$ refer to the director, chirality, polarization, and rotational axial (electric toroidal) vector, respectively, and ' refers to the time-reversal broken version. All left-hand-side $1 \mathrm{D}$ objects $\left(\mathscr{D}, \mathscr{C}, \mathscr{D}^{\prime}, \mathscr{C}^{\prime}\right)$ in Fig. 1 have not-broken $\mathbf{R}$ (i.e., they are director-like) and all righthand-side $1 D$ objects $\left(\mathscr{P}, \mathscr{R}, \mathscr{P}^{\prime}, \mathscr{R}^{\prime}\right)$ have broken $\mathbf{R}$ (i.e., they are vector-like).

\section{RESULTS AND DISCUSSION}

\section{Eight types of 1D objects}

We have attempted to identify various exemplary 1D objects that show SOS with each of the eight Hlinka's classifications ${ }^{4}$, which are listed in Fig. 1 and described below:

$\mathscr{D}$ (no broken symmetry): director. Simple antiferromagnetic order in a chain, up-up-down-down antiferromagnetic order in a chain, and up-up-down-down antiferromagnetic order in a chain with two kinds of bonding. For example, the magnetic point group $\mathrm{mmm1}^{\prime}$ (\#8.2.25) has I, R, R, M, M, T, so fit the criteria for $\mathscr{D}$.

$\mathscr{C}$ (broken $\{\mathbf{I}, \mathbf{M}, \underline{\mathbf{M}\}}\}$ ): chirality. Crystallographic chirality, helical spins, a magnetic toroidal moment with the out-of-plane canted moment, a magnetic quadrupole moment with alternating out-ofplane canted moments, linear momentum (velocity) with spin or

\footnotetext{
1Department of Physics and Astronomy, Rutgers Center for Emergent Materials, Piscataway, NJ, USA. ${ }^{凶}$ email: sangc@physics.rutgers.edu
} 


\section{Eight types of $1 D$ objects}

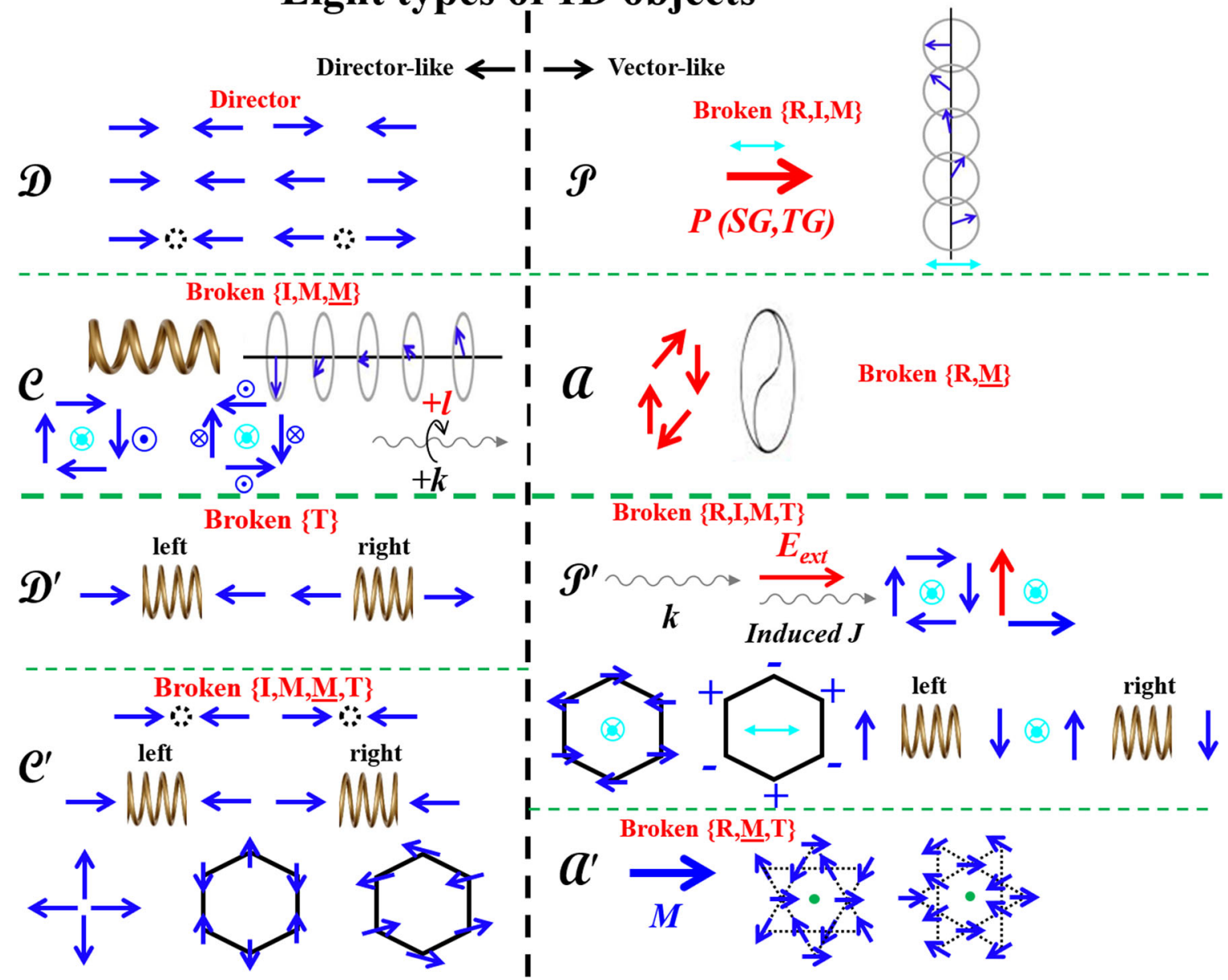

Fig. 1 Eight types of 1D objects. Each type has a particular set of broken symmetries: $\mathscr{D} ;$ no broken symmetry, $\mathscr{C}$; broken $\{\mathbf{I}, \mathbf{M}, \mathbf{M}\}, \mathscr{D} ;$; broken $\{\mathbf{T}\}, \mathscr{C}^{\prime}$; broken $\{\mathbf{I}, \mathbf{M}, \mathbf{M}, \mathbf{T}\}, \mathscr{P}$; broken $\{\mathbf{R}, \mathbf{I}, \mathbf{M}\}, \boldsymbol{a}$; broken $\{\mathbf{R}, \mathbf{M}\}, \mathscr{P}^{\prime}$; broken $\{\mathbf{R}, \mathbf{I}, \mathbf{M}, \mathbf{T}\}, \boldsymbol{a}^{\prime}$; broken $\{\mathbf{R}, \mathbf{M}, \mathbf{T}\}$. When $\{\mathbf{T}\}$ is additionally broken, then $\mathscr{D}, \mathscr{C}, \mathscr{P}$, and $\overline{\boldsymbol{a}}$ become $\mathscr{D}^{\prime}, \mathscr{C}^{\prime}, \mathscr{P}^{\prime}, \boldsymbol{a}^{\prime}$, respectively. Note that all left-hand-side $1 \mathrm{D}$ objects $\left(\overline{\mathscr{D}}^{\prime}, \mathscr{C}, \mathscr{D}^{\prime}, \mathscr{C}^{\prime}\right)$ have not-broken $\{\mathbf{R}\}$, so they are director-like, and all right-hand-side $1 \mathrm{D}$ objects $\left(\mathscr{P}, \mathscr{R}, \mathscr{P}^{\prime}, \mathscr{R}^{\prime}\right)$ have broken $\{\mathbf{R}\}$, so they are vector-like. A large number of quasi-1D schematic examples are also shown: blue arrows are spins or magnetization, red arrows are polarizations or electric fields, wiggly arrows are velocity vectors or linear momenta, " $+\boldsymbol{l}$ " represents angular momentum or circular polarization, light-blue symbols depict the reference $1 \mathrm{D}$ direction, green dots are inversion centers, dashed black circles are anions, and golden springs represent mono-axial chiral objects. The reference 1D direction is the horizontal direction on the page plane, except when there is an explicit light-blue symbol depicting the 1D direction.

orbital angular momentum along the linear momentum direction, circularly-polarized light, and vortex beam having orbital angular momentum along the vortex beam direction. For example, the point groups 222, 32, 422, 622, and 23 fit the criteria of broken $\{\mathbf{I}, \mathbf{M}, \mathbf{M}\}$.

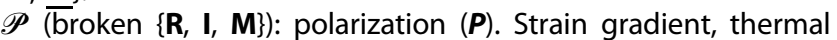
gradient, and cycloidal spins. For example, the point groups $2 \mathrm{~mm}$, $3 \mathrm{~m}, 4 \mathrm{~mm}$, and $6 \mathrm{~mm}$ fit the criteria of broken $\{\mathbf{R}, \mathbf{I}, \mathbf{M}\}$.

$\boldsymbol{a}$ (broken $\{\mathbf{R}, \mathbf{M}\}$ ): axial vector of ferro-rotation. Crystallographic rotational distortions, and electric toroidal moment. For example, the point groups $\overline{1}, \overline{3}, \overline{4}, \overline{6}, 2 / \mathrm{m}, 4 / \mathrm{m}$, and $6 / \mathrm{m}$ fit the criteria of broken $\{\mathbf{R}, \mathbf{M}\}$.

$\mathscr{D}^{\prime}$ (broken $\{\mathbf{T}\}$ ): a director with broken $\{\mathbf{T}\}$. Up-up-down-down antiferromagnetic order in a chain with alternating two kinds of chiral bonding environments. For example, the magnetic point group mmm (\#8.1.24) has I, R, R, M, M, so fit the criteria for $\mathscr{D}^{\prime}$.

$\mathscr{C}^{\prime}$ (broken $\left.\{\mathbf{I}, \mathbf{M}, \mathbf{M}, \mathbf{T}\}\right)$ : $\bar{c}$ chirality with broken $\{\mathbf{T}\}$. Simple antiferromagnetic order in a chain with two kinds of bonding, simple antiferromagnetic order in a chain with alternating two kinds of chiral bonding environments, which corresponds to the magnetic state of $\mathrm{Cr}_{2} \mathrm{O}_{3}$, magnetic monopole, an in-plane antiferromagnetic order on the honeycomb lattice, and an in-plane antiferromagnetic order with in-plane canting on the honeycomb lattice. For example, the magnetic point groups $\overline{3}^{\prime} \mathrm{m}^{\prime}(\# 20.4 .74)$ and $6 \mathrm{~m}^{\prime} \mathrm{m}^{\prime}$ (\#25.4.94) fit the criteria of broken $\{\mathbf{I}, \mathbf{M}, \mathbf{M}, \mathbf{T}\}$.

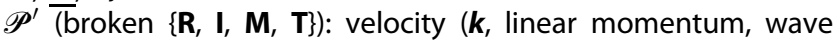
vector). The act of applying electric field inducing electric current, magnetic toroidal moments, an in-plane antiferromagnetic order on a honeycomb lattice, Ising antiferromagnetic order on a honeycomb lattice, and antiferromagnetic order in a chain with alternating two kinds of chiral bonding environments, corresponding to the spin-flopped state of $\mathrm{Cr}_{2} \mathrm{O}_{3}$. Note that all specimen constituents with the $\mathscr{P}^{\prime}$ character can exhibit non-reciprocal directional dichroism. For example, the magnetic point group $6 \mathrm{~mm}^{\prime}$ ' (\#25.2.92) fits the criteria of broken $\{\mathbf{R}, \mathbf{I}, \mathbf{M}, \mathbf{T}\}$.

$\boldsymbol{a}^{\prime}$ (broken $\{\mathbf{R}, \mathbf{M}, \mathbf{T}\}$ ): axial-vector with broken $\{\mathbf{T}\}$. Magnetization $(\boldsymbol{M})$, and two kinds of magnetic order in kagome lattice having SOS with $\boldsymbol{M}$. For example, the magnetic point groups $\overline{3} \mathrm{~m}^{\prime}$ (\#20.5.75) fits the criteria of broken $\{\mathbf{R}, \mathbf{M}, \mathbf{T}\}$. The magnetic point groups $2^{\prime} / \mathrm{m}^{\prime}$ (5.5.16) and $\overline{1}(\# 2.1 .3)$, which correspond to the magnetic states of $\mathrm{a}-\mathrm{Fe}_{2} \mathrm{O}_{3}$ above and below the Morin transition, respectively, have broken $\{\mathbf{R}, \mathbf{R}, \mathbf{M}, \mathbf{M}, \mathbf{T}\}$, so does have SOS with $\boldsymbol{a}^{\prime}$. 
(a)
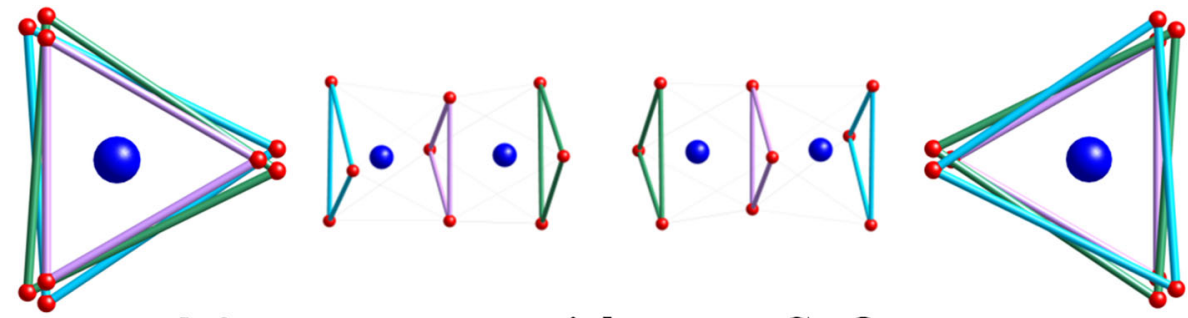

left

right

$\mathrm{Cr}_{2} \mathrm{O}_{3}$

(b)

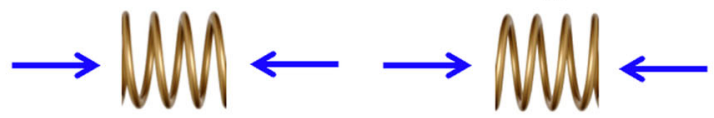

\title{
Broken $\{\underline{\mathbf{M}}, \mathbf{M} \otimes \mathbf{R}, \mathbf{T}\}$ MOKE
}

\author{
left
}

right

(c)
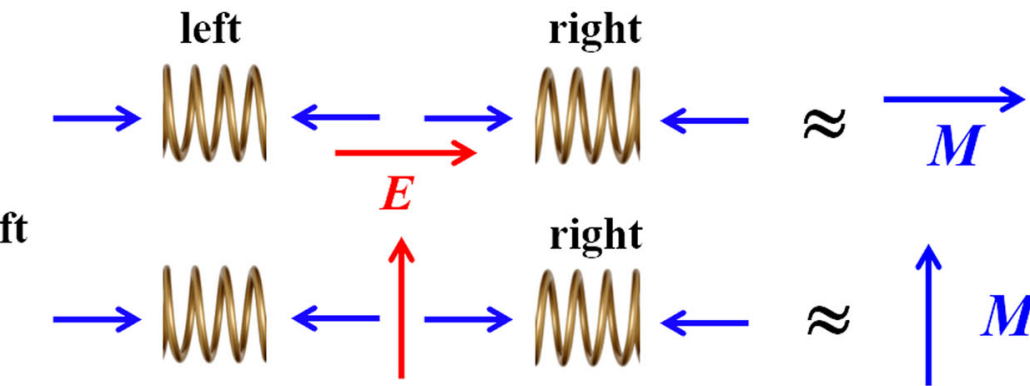

(d)

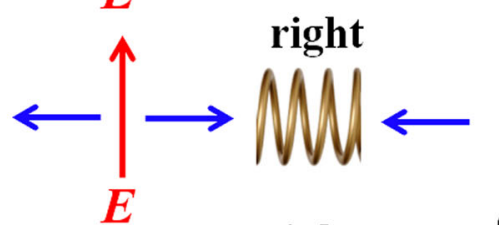<smiles>C=CC</smiles>

left

(e)
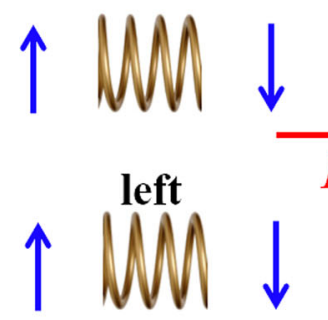

right<smiles>[AlH2]</smiles>

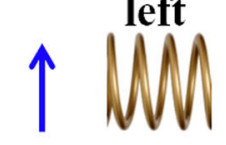

left

(g)

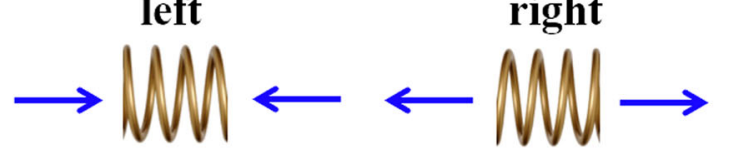

left

(h)
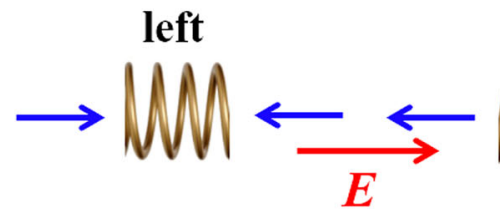

right

\section{.}

right

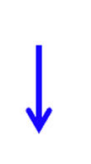

$\mathrm{Cr}_{2} \mathrm{O}_{3}$ above spin-flop transition

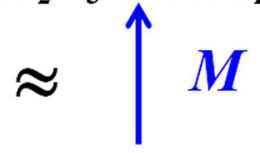

Similar with

$\mathrm{Fe}_{2} \mathrm{O}_{3}$ below Morin transition

$\mathrm{Fe}_{2} \mathrm{O}_{3}$ above Morin transition.

$\approx$

$\underset{M}{ }$ Broken $\{\mathrm{R}, \underline{\mathrm{M}}, \mathrm{T}\}$

(i)

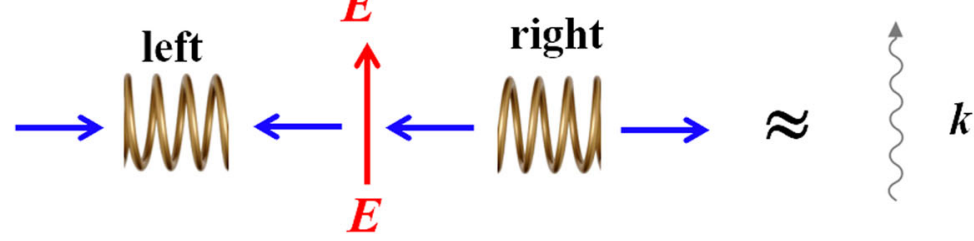

Fig. 2 Various magnetic states of $\mathrm{Cr}_{2} \mathrm{O}_{3}$ or $\mathbf{a}-\mathrm{Fe}_{2} \mathrm{O}_{3}$, and their SOS relationships. a Crystallographic structure of a c-direction chain of $\mathrm{Cr}_{2} \mathrm{O}_{3}$ or $\alpha-\mathrm{Fe}_{2} \mathrm{O}_{3}$, and left-chiral and right-chiral arrangements of three oxygen tringles are shown in the left-hand-side and right-hand-side projections along the $\mathrm{c}$ axis, respectively. $\mathbf{b}$ The magnetic state of $\mathrm{Cr}_{2} \mathrm{O}_{3}$ with broken $\{\mathbf{M}, \mathbf{M} \otimes \mathbf{R}$, T $\}$, so can exhibit MOKE. $\mathbf{c}$, $\mathbf{d}$ The magnetic state of $\mathrm{Cr}_{2} \mathrm{O}_{3}$ in $\boldsymbol{E}$ shows SOS with $\boldsymbol{M}$. e The spin-flopped state of $\mathrm{Cr}_{2} \mathrm{O}_{3}$ in $\boldsymbol{E}$ shows SOS with $\boldsymbol{M}$. $\mathbf{f}$ The magnetic state of $\alpha-\mathrm{Fe}_{2} \mathrm{O}_{3}$ above the Morin transition has SOS with $\boldsymbol{M}$. $\mathbf{g}$ This magnetic state has no SOS with any $\boldsymbol{M}$, and is somewhat similar with the magnetic state of $\alpha$-Fe ${ }_{2} \mathrm{O}_{3}$ below the Morin transition. However, the magnetic state of $\alpha-\mathrm{Fe}_{2} \mathrm{O}_{3}$ below the Morin transition has lower symmetries $(\overline{1})$, and turns out to have SOS with $\boldsymbol{M}$ in any arbitrary direction. $\mathbf{h}$, $\mathbf{i}$ The magnetic state of $\alpha-\mathrm{Fe}_{2} \mathrm{O}_{3}$ below the Morin transition in $\boldsymbol{E}$ has $\mathrm{SOS}$ with $\boldsymbol{k}$.

As exemplary cases, we, first, discuss how our SOS approach can be helpful to understand the physics of $\mathrm{Cr}_{2} \mathrm{O}_{3}$ with linear magnetoelectricity and $\mathrm{a}-\mathrm{Fe}_{2} \mathrm{O}_{3}$ with a canted magnetic moment and also to predict a new phenomenon in $\mathrm{a}-\mathrm{Fe}_{2} \mathrm{O}_{3}$. In both $\mathrm{Cr}_{2} \mathrm{O}_{3}$ and $\mathrm{a}-\mathrm{Fe}_{2} \mathrm{O}_{3}$, the 3D lattice consists of a simple hexagonal stacking of $1 \mathrm{D} c$-direction chains of magnetic ions with alternating left-right chiral bonding environments, as shown in Fig. 2a. Magnetic orders in $\mathrm{Cr}_{2} \mathrm{O}_{3}$ and $\mathrm{a}-\mathrm{Fe}_{2} \mathrm{O}_{3}$ above the Morin transition are schematically 

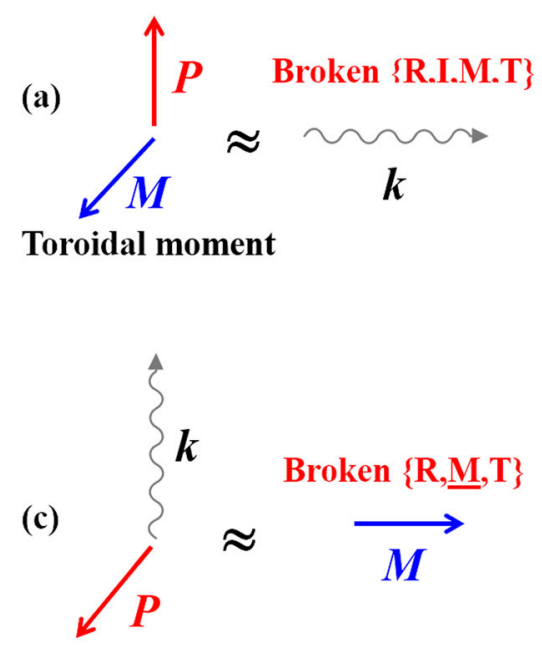

Fig. 3 Three permutable SOS among $\boldsymbol{\alpha}^{\prime}, \mathscr{P}$, and $\mathscr{P}^{\prime}$ and SOS relationships with $\boldsymbol{\alpha}$. Magnetization (M or magnetic field $\left.\boldsymbol{H}\right)$, polarization $(\boldsymbol{P}$ or electric field $\boldsymbol{E})$, and velocity $(\boldsymbol{k})$ are representative for $\boldsymbol{\boldsymbol { A }}^{\prime}, \mathscr{P}$, and $\mathscr{P}^{\prime}$, respectively. a $\boldsymbol{P} \times \boldsymbol{M}$ has broken $\{\mathbf{R}, \mathbf{I}, \mathbf{M}, \mathbf{T}\}$ along the horizontal direction in the page plane, so has SOS with $\boldsymbol{k}$. $\mathbf{b} \boldsymbol{M} \times \boldsymbol{k}$ has broken $\{\mathbf{R}, \mathbf{I}, \mathbf{M}\}$ along the horizontal direction, so has SOS with $\boldsymbol{P}$. c $\boldsymbol{k} \times \mathbf{P}$ has broken $\{\mathbf{R}, \mathbf{M}, \mathbf{T}\}$ along the horizontal direction, so has SOS with $\boldsymbol{M}$. $\mathbf{d} \boldsymbol{E} \times \boldsymbol{\boldsymbol { \alpha }}$ has broken $\{\mathbf{R}, \mathbf{I}, \mathbf{M}\}$ along the horizontal direction, so has SOS with $\boldsymbol{P}, \boldsymbol{H} \times \boldsymbol{\alpha}$ has broken $\{\mathbf{R}, \mathbf{M}, \mathbf{T}\}$ along the horizontal direction, so has SOS with $\boldsymbol{M}$, and $\boldsymbol{k} \times \boldsymbol{\alpha}$ has broken $\{\mathbf{R}, \mathbf{I}, \mathbf{M}, \mathbf{T}\}$ along the horizontal direction, so has SOS with $\boldsymbol{k}$.

shown in Fig. 2b, f, respectively, so they correspond to $\mathscr{C}^{\prime}$ and $\boldsymbol{a}^{\prime}$, respectively. In the case of $\mathrm{Cr}_{2} \mathrm{O}_{3}$, the magnetic state in Fig. $2 \mathrm{~b}$ has broken $\{\mathbf{I}, \mathbf{M}, \mathbf{M}, \mathbf{T}\}$, and we can construct an SOS relationship by adding further broken symmetries such as broken $\{\mathbf{R}, \mathbf{I}, \mathbf{M}\}$ of $\boldsymbol{P}$ or electric field. As shown in Fig. 2c, d, the magnetic state of $\mathrm{Cr}_{2} \mathrm{O}_{3}$ in the presence of an electric field does have SOS with $\boldsymbol{M}$, so it shows a diagonal linear magnetoelectric effect ${ }^{5}$. Applying a large magnetic field along the $c$ axis induces a spin-flopped state, shown in Fig. 2e, and this state exhibits an off-diagonal linear magnetoelectric effect, as the SOS in Fig. 2e indicates. The canted magnetic state of $\mathrm{a}-\mathrm{Fe}_{2} \mathrm{O}_{3}$ above the Morin transition is consistent with the SOS in Fig. 2f. The magnetic state in Fig. $2 \mathrm{~g}$ has no SOS with any $\boldsymbol{M}$, and is somewhat similar with the magnetic state of $\mathrm{a}-\mathrm{Fe}_{2} \mathrm{O}_{3}$ below the Morin transition. However, the magnetic state of $\mathrm{a}-\mathrm{Fe}_{2} \mathrm{O}_{3}$ below the Morin transition has lower symmetries $(\overline{1})$, and turns out to have SOS with $\boldsymbol{M}$ in any arbitrary direction. Note that the schematic in Fig. $2 b$ has broken $\{\mathbf{M}, \mathbf{M} \otimes \mathbf{R}, \mathbf{T}\}$, so is supposed to exhibit a magnetooptical Kerr effect (MOKE), and indeed, does show non-zero MOKE in $\mathrm{Cr}_{2} \mathrm{O}_{3}{ }^{2,6}$. It is very interesting to notice that the magnetic state of $\mathrm{a}-\mathrm{Fe}_{2} \mathrm{O}_{3}$ below the Morin transition in the presence of an electric field does show SOS with $\boldsymbol{k}$, which means that it can exhibit a nonreciprocal effect such as an optical-diode effect, which can be experimentally confirmed in the future.

We emphasize that $\mathscr{D}, \mathscr{C}, \mathscr{P}, \boldsymbol{\alpha}, \mathscr{D}^{\prime}, \mathscr{C}^{\prime}, \mathscr{P}^{\prime}$, and $\boldsymbol{\alpha}^{\prime}$ are well-defined and all distinct, and when a specimen constituent has SOS with one of $\left(\mathscr{D}, \mathscr{C}, \mathscr{P}, \boldsymbol{a}, \mathscr{D}^{\prime}, \mathscr{C}^{\prime}, \mathscr{P}^{\prime}, \boldsymbol{a}^{\prime}\right)$, the specimen constituent can have more (but not less) broken symmetries than the one. We also note that, for example, broken $\{\mathbf{R}, \underline{\mathbf{M}}, \mathbf{T}\}$ for $\boldsymbol{M}$ (or $\boldsymbol{a}^{\prime}$ ) lists all "independently broken" symmetries, so, $\mathbf{I}$ is not broken and consequently $\mathbf{I} \otimes \mathbf{T}$ is broken for $\boldsymbol{M}$ (or $\boldsymbol{a}^{\prime}$ ). Thus, I is not broken in $\boldsymbol{M}$ (or $\boldsymbol{a}^{\prime}$ ), but some real 3D ferromagnets, having SOS with $\boldsymbol{M}$ (or $\boldsymbol{a}^{\prime}$ ), can have broken $\mathbf{I}$. However, no ferromagnets can have higher symmetries than $\boldsymbol{M}$ (or $\boldsymbol{a}^{\prime}$ ). Thus, all ferromagnets do have also broken $\mathbf{I} \otimes \mathbf{T}$ since $\mathbf{I} \otimes \mathbf{T}$ is broken in $\boldsymbol{M}$ (or $\boldsymbol{\alpha}^{\prime}$ ). In other words, real 3D ferromagnets can have all broken $\mathbf{I}, \mathbf{T}$, and $\mathbf{I} \otimes \mathbf{T}$. Thus, the necessary and sufficient condition for ferromagnetism or a non-zero net magnetic moment in real 3D materials is having SOS with $\boldsymbol{M}$ (or $\boldsymbol{a}^{\prime}$ ) having broken $\{\mathbf{R}, \underline{\mathbf{M}}, \mathbf{T}\}$.

\section{Permutable SOS of $\mathscr{P} \times \mathscr{P}^{\prime} \approx \boldsymbol{a}^{\prime}$}

The usefulness of the SOS approach is that it can be commonly applied to various physical quantities that share the same set of broken symmetries. $\mathscr{P}$ can be polarization $(\boldsymbol{P})$ or an electric field $(\boldsymbol{E})$, but it can be also a temperature gradient, strain gradient, or surface effective electric field, $\mathscr{P}^{\prime}$ can be velocity/wave vector (k) of electric current, spin-wave or thermal current, or toroidal moment, and $\boldsymbol{A}^{\prime}$ is magnetization $(\boldsymbol{M})$ or magnetic field $(\boldsymbol{H})$. Moreover, the SOS relationships established among them can sometimes be permutable. Figure $3 a-c$ shows pictorially the permutable SOS relationships of $\mathscr{P} \times \boldsymbol{a}^{\prime} \approx \mathscr{P}^{\prime}, \boldsymbol{a}^{\prime} \times \mathscr{P}^{\prime} \approx$ $\mathscr{P}, \mathscr{P}^{\prime} \times \mathscr{P} \approx \boldsymbol{\alpha}^{\prime}$, respectively. Note that all of $\mathscr{P}, \mathscr{P}^{\prime}$, and $\boldsymbol{\alpha}^{\prime}$ are vector-like, i.e., not director-like.

It turns out that the motion of quasiparticles such as electrons, phonons, magnons, and photons in a specimen or the motion of the specimen itself can be non-reciprocal if the specimen constituent has SOS with $\boldsymbol{k}$ of the motion. If a symmetry operation links $+\boldsymbol{k}$ and $-\boldsymbol{k}$, and a specimen constituent where quasiparticles are moving with $\pm \boldsymbol{k}$ is invariant under the symmetry operation, then the experiment becomes reciprocal, i.e., there is no difference between the experimental results with $+\boldsymbol{k}$ and those with $-\boldsymbol{k}$. On the other hand, when a specimen constituent has SOS with $\boldsymbol{k}$, then there is no symmetry operation that can link these two experiments: a specimen constituent with $+\boldsymbol{k}$ and the identical specimen with $-\boldsymbol{k}$. Thus, the experiments can be non-reciprocal. Note that the magnitude of non-reciprocal effects cannot be estimated from symmetry considerations alone, and this estimation requires an understanding of the microscopic mechanism of the non-reciprocal effects. Thus, Fig. 3a means that if $\boldsymbol{H}$ is applied to any polar magnets, then the electron, light, phonons, or spin-wave propagation can be non-reciprocal, i.e., exhibit directional dichroism. Indeed, for example, diode-like light propagation has been observed in polar $(\mathrm{Fe}, \mathrm{Zn})_{2} \mathrm{Mo}_{3} \mathrm{O}_{8}$ in $\boldsymbol{H}^{7}$, and electron transport in polar $\mathrm{BiTeBr}^{8}$ in $\boldsymbol{H}$ turns out to be nonreciprocal in experimental configurations consistent with Fig. 3a. We anticipate that applying in-plane $\boldsymbol{H}$ to polar $\mathrm{Fe}(\mathrm{Mn}, \mathrm{Ni}, \mathrm{Zn})_{2} \mathrm{Mo}_{3} \mathrm{O}_{8}$ or polar (and chiral) $\mathrm{Ni}_{3} \mathrm{TeO}_{6}{ }^{9}$ will induce a non-reciprocal spin-wave along the in-plane direction perpendicular to $\boldsymbol{H}$.

Figure $3 b$ represents all kinds of (anomalous) Hall-type effects (Hall, Ettingshausen, Nernst, and thermal Hall). Here, ( $\boldsymbol{k}$ and $\boldsymbol{P}$ ) represents (electric current and induced voltage), (electric current and induced thermal gradient), (thermal gradient and induced 
2H-MoS, 2 under tensile strain

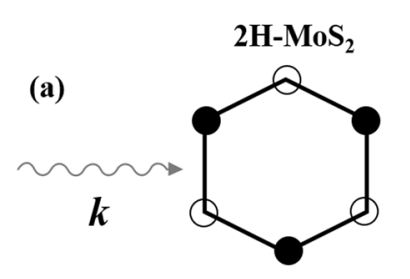

(1)

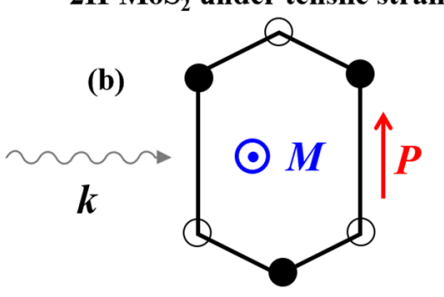

2H-MoS 2 under compressive strain

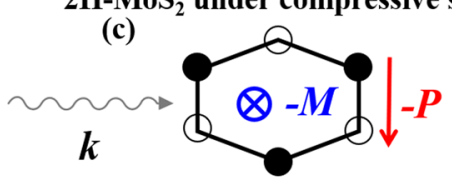

(d)<smiles>CCCCC12C3C4C5C3C1C5C42</smiles>

(e)

$\mathrm{Td}-\mathrm{Mo}(\mathrm{W}) \mathrm{Te}_{2}$
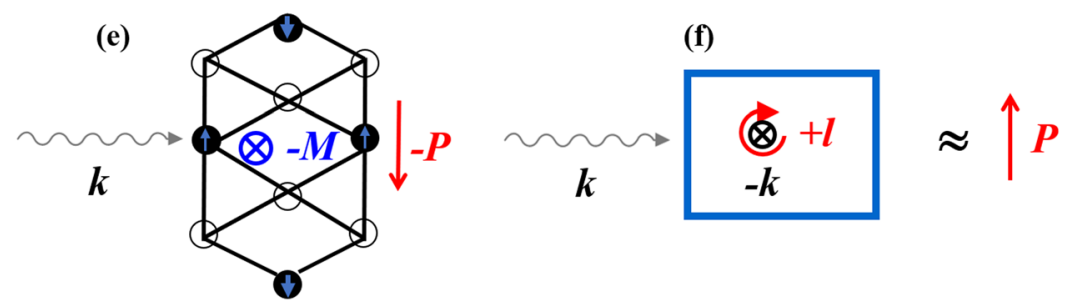

(g)

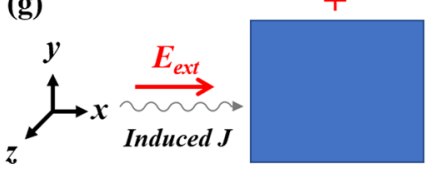

(h)

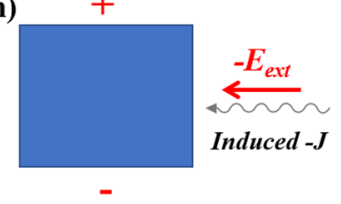

(i)

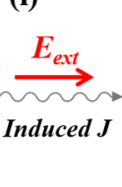

(j)

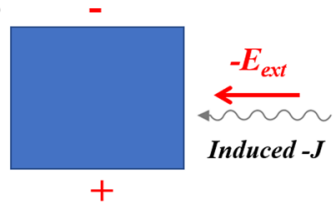

Fig. 4 Current-induced $\boldsymbol{M}$, circular dichroism Hall effect (CDHE), and non-linear Hall effect (NLHE). a-c Monolayer of 2H-MoS $S_{2}$ : (a); no strain, (b); uni-directional uniform tensile strain along the vertical direction on the page plane, and (c); uni-directional uniform compressive strain along the vertical direction on the page plane. $\mathbf{d}$, e show monolayer $1 \mathrm{~T}-\mathrm{Mo}(\mathrm{W}) \mathrm{Te}_{2}$ and $\mathrm{T}_{\mathrm{d}}-\mathrm{Mo}(\mathrm{W}) \mathrm{Te}_{2}$, respectively. Closed black circles, darkblue arrows, open black circles, wiggly arrows, red arrows, and blue symbols represent cations, cation distortions, anions, uniform electric currents, polarizations, and induced magnetizations, respectively. Thus, (b), (c), and (e) represent the current-induced magnetization in polar conductors. This current-induced magnetization is directly related to the presence of non-linear Hall effects in polar conductors without any external magnetic field. $\mathbf{f}$ depicts the SOS relationship for CDHE with the electric current flow with $\boldsymbol{k}$, circular-light illumination with $-\boldsymbol{k}$ and $+\boldsymbol{I}$, and $\boldsymbol{P}$ representing the induced Hall voltage. $\mathbf{g}$-j Four possible configurations of Hall-type voltages induced by electric currents in external electric fields.

voltage), and (thermal gradient and induced thermal gradient) for the Hall, Ettingshausen, Nernst, and thermal Hall effects, respectively. Figure $3 \mathrm{c}$ can also represent numerous physical phenomena, including the off-diagonal linear magnetoelectric effect in $\mathrm{Cr}_{2} \mathrm{O}_{3}$ in the spin-flopped state (Fig. 2e), the spin Hall effect, the spin Nernst (or thermal spin Hall) effect, currentinduced magnetization in polar conductors, and also non-linear Hall effects in polar conductors ${ }^{10}$. Note that $\boldsymbol{\alpha}^{\prime} \times \mathscr{P}^{\prime} \approx \mathscr{P}$ and $\mathscr{P}^{\prime} \times \mathscr{P} \approx \boldsymbol{a}^{\prime}$ indicate that all magnetic states having SOS with $\mathscr{P}^{\prime}$ should exhibit off-diagonal linear magnetoelectric effects. When $\boldsymbol{P}$ represents the surface effective electric field, electric current and thermal current for $\boldsymbol{k}$ can induce spin current flow along the direction perpendicular to $\boldsymbol{P}$ and the current directionthe resulting surface magnetization is represented by $\boldsymbol{M}$. Here, the electric current and the thermal current correspond to the spin Hall effect and the spin Nernst (or thermal spin Hall) effect, respectively. Figure $3 \mathrm{c}$ also represents that electric current, thermal current, or light propagation along a direction perpendicular to $\boldsymbol{P}$ in polar materials can induce $\boldsymbol{M}$ along the direction perpendicular to both the $\boldsymbol{P}$ and the current/light propagation directions. Even though $\boldsymbol{M}$ in polar materials induced by thermal current/light has not been reported, $\boldsymbol{M}$ in polar conductors induced by electric current has been reported, interestingly in an exotic material of strained monolayer $\mathrm{MoS}_{2}^{11,12}$. It will be interesting to observe this current-induced $\boldsymbol{M}$ in bulk nonmagnetic polar conductors such as $\mathrm{Ca}_{3} \mathrm{Ru}_{2} \mathrm{O}_{7}, \mathrm{GeTe}, \mathrm{TaAs}$, and $\mathrm{BiTel}(\mathrm{Br}, \mathrm{Cl})^{13-16}$. In addition, it will be fundamentally important to observe $\boldsymbol{M}$ in polar materials induced by thermal current or lightin this case, the polar materials do not have to be electric conductors, in principle.
The monolayer of $2 \mathrm{H}-\mathrm{MoS}_{2}$, shown in Fig. $4 \mathrm{a}$, is non-centrosymmetric, but non-polar due to the presence of $C_{3}$ symmetry. However, uniaxial uniform strain along a particular in-plane direction breaks the $C_{3}$ symmetry and induces $\boldsymbol{P}$ in the direction perpendicular to the strain. When an electric current is applied to an in-plane direction perpendicular to the strain-induced $\boldsymbol{P}$, then $\boldsymbol{M}$ develops along the out-of-plane direction, which is depicted in Fig. 4b, c. Tensile and compressive strains result in opposite Ms. A similar situation, but without external strain, should also work for monolayer $\mathrm{T}_{d}-\mathrm{WTe} \mathrm{e}_{2}$ or $\mathrm{T}_{d}-\mathrm{MoTe}_{2}$. Monolayer $\mathrm{T}_{d}-\mathrm{WTe} \mathrm{e}_{2}$ or $\mathrm{T}_{\mathrm{d}}-\mathrm{MoTe}_{2}$ with W/Mo zigzags does have in-plane polarization perpendicular to the zigzag direction. When an electric current is applied along the zigzag direction, $\boldsymbol{M}$ along the out-of-plane direction should be induced, which requires future experimental confirmation. In fact, this $\boldsymbol{M}$ induced by electric current in polar conductors is directly related to the presence of the non-linear Hall effect that we will discuss next.

First, we attempt to figure out the requirement for quadratictype non-linear Hall effect (NLHE) without any applied magnetic field. We consider four Fig. $4 \mathrm{~g}-\mathrm{j}$ situations of Hall-type voltages with an applied external electric field $\left(+-\boldsymbol{E}_{\text {ext }}\right)$. To discuss the 3D arrangements of NLHE measurements, we will use these notations: $\mathbf{R}_{\mathbf{x}}=$ twofold rotation operation around the $x$-axis, $\mathbf{M}_{\mathbf{x}}=$ mirror operation with the mirror perpendicular to the $x$-axis, etc. Any of $\left\{\mathbf{R}_{\mathbf{y}}, \mathbf{M}_{\mathbf{x}}, \mathbf{M}_{\mathbf{z}}, \mathbf{T}\right\}$ links between Fig. $4 \mathrm{~g}$, $\mathrm{h}$ (or, (g) or (h) itself). NLHE should not depend on the sign of the external $\boldsymbol{E}$, so NLHE may require not-broken $\left\{\mathbf{R}_{\mathbf{y}}, \mathbf{M}_{\mathbf{x}}, \mathbf{M}_{\mathbf{z}}, \mathbf{T}\right\}$. However, breaking any of $\left\{\mathbf{R}_{\mathbf{y}}\right.$, $\mathbf{M}_{\mathbf{x}}, \mathbf{M}_{\mathbf{z}}, \mathbf{T}$ \} does not make NLHE vanish. Now, any cross-link between Fig. $4 \mathrm{~g} / \mathrm{h}$, i/j can happen with $\left\{\mathbf{R}_{\mathbf{x}}, \mathbf{R}_{\mathbf{z}}, \mathbf{I}, \mathbf{M}_{\mathbf{y}}\right\}$, so not-broken any of $\left\{\mathbf{R}_{\mathbf{x}}, \mathbf{R}_{\mathbf{z}}, \mathbf{I}, \mathbf{M}_{\mathbf{y}}\right\}$ will lead to NLHE to vanish. Therefore, broken $\left\{\mathbf{R}_{\mathbf{x}}, \mathbf{R}_{\mathbf{z}}, \mathbf{I}, \mathbf{M}_{\mathbf{y}}\right\}$ is required to have non-zero NLHE. $\left\{\mathbf{R}_{\mathbf{x}}, \mathbf{R}_{\mathbf{z}}, \mathbf{I}, \mathbf{M}_{\mathbf{y}}\right\}$ 
becomes $\{\mathbf{R}, \mathbf{I}, \mathbf{M}\}$ with the respect to the vertical reference direction on the page plane (i.e., the $y$-axis). Thus, broken $\{\mathbf{R}, \mathbf{I}, \mathbf{M}\}$, more precisely speaking broken $\left\{\mathbf{R}_{\mathbf{x}}, \mathbf{R}_{\mathbf{z}}, \mathbf{I}, \mathbf{M}_{\mathbf{y}}\right\}$, is the necessary and sufficient condition for non-zero NLHE. $\boldsymbol{P}$ has broken $\{\mathbf{R}, \mathbf{I}, \mathbf{M}\}$ and also not-broken $\left\{\mathbf{R}_{\mathbf{y}}, \mathbf{M}_{\mathbf{x}}, \mathbf{M}_{\mathbf{z}}, \mathbf{T}\right\}$, but in principle, any specimen constituents having SOS with $\boldsymbol{P}$ can exhibit non-zero NLHE. In other words, the specimen constituents with non-zero NLHE can have lower symmetry than $\boldsymbol{P}$ (i.e., can have broken some of $\left\{\mathbf{R}_{\mathbf{y}}, \mathbf{M}_{\mathbf{x}}\right.$ $\left.\left.\mathbf{M}_{\mathbf{z}}, \mathbf{T}\right\}\right)$. Note that in principle, broken $\left\{\mathbf{R}_{\mathbf{x}}, \mathbf{R}_{\mathbf{z}}, \mathbf{I}, \mathbf{M}_{\mathbf{y}}\right\}$ can be achieved in any of non-centrosymmetric materials when the $x, y$, and $z$ axes are properly chosen, even though the magnitudes of NLHE maybe not significant when the $x, y$, and $z$ axes are not along with the principal crystallographic directions.

Now, there exists a straightforward but elegant relationship between current-induced magnetization and NLHE in polar conductors. When an electric current, $\boldsymbol{J}$, is applied to a polar conductor along a direction perpendicular to $\boldsymbol{P}, \boldsymbol{M}$ is induced along a direction perpendicular to both $\boldsymbol{J}$ and $\boldsymbol{P}$. This, induced $\boldsymbol{M}$ acts like a magnetic field for the standard Hall effect, and Hall voltage along the $\boldsymbol{P}$ direction goes like $\boldsymbol{J} \times \boldsymbol{M} \propto \boldsymbol{J}^{\mathbf{2}}$, which explains quadratic-type non-linear Hall effect in polar conductors. Therefore, strained monolayer $\mathrm{MoS}_{2}$ should exhibit NLHE along the strain-induced $\boldsymbol{P}$ direction. In fact, the observation of NLHE in ultrathin $\mathrm{WTe}_{2}$ may be consistent with the above discussion ${ }^{17}$. Note that bulk non-magnetic polar conductors such as $\mathrm{Ca}_{3} \mathrm{Ru}_{2} \mathrm{O}_{7}$, GeTe, TaAs, and $\mathrm{BiTel}(\mathrm{Br}, \mathrm{Cl})$ should exhibit NLHE, in addition to current-induced $\boldsymbol{M}$.

We also note that the so-called circular dichroism Hall effect can be understood on a similar footing ${ }^{18}$. Illumination of circularlypolarized light on a chiral material can induce an electric current, which is called a circular photogalvanic effect (CPGE) $)^{19-21}$. In the case of CPGE, one can consider if or not the entire experimental setup with induced current changes in a systematic manner under all symmetry operations-the so-called symmetry operational systematics (SOSs). It turns out that in terms of SOSs, a similar phenomenon can occur in a material with a net magnetic moment, rather than chirality ${ }^{1}$. Now, with the consideration of SOSs, one can also show that illumination of circularly-polarized light on any materials can induce $\boldsymbol{M}$. This circularly-polarized lightinduced $\boldsymbol{M}$ can act as a magnetic field, so when electric current flows along a direction perpendicular to the induced $\boldsymbol{M}$, then a Hall voltage can develop along the direction perpendicular to both $\boldsymbol{M}$ and current without any applied magnetic field, which is called a circular dichroism Hall effect (CDHE). Therefore, in principle, this CDHE can occur in any materials where electric current flow is possible. This CDHE can be also understood in terms of the SOS relationship in Fig. 4f. Emphasize that the specimen showing with a blue rectangle appears to have no broken symmetries, but the SOS relationship still holds even if the specimen has any broken symmetries by the definition of an SOS relationship. This again guarantees that CDHE can occur in any materials where electric current flow is possible. In addition, the electric current flow can be replaced by the thermal current, and also $\boldsymbol{P}$ can stand for a thermal gradient, rather than a Hall voltage. Therefore, there can exist Ettingshausen, Nernst, or thermal Hall effect versions of CDHE in any materials with circularly-polarized light (or vortex beam with orbital angular momentum) illumination without any applied magnetic field.

We would like to discuss three more cross-product-type SOS relationships: $\mathscr{P} \times \boldsymbol{a} \approx \mathscr{P}, \boldsymbol{a}^{\prime} \times \boldsymbol{a} \approx \boldsymbol{a}^{\prime}$, and $\mathscr{P}^{\prime} \times \boldsymbol{a} \approx \mathscr{P}^{\prime}$, which are illustrated in Fig. 3d. Note that all of $\mathscr{P}, \boldsymbol{\alpha}, \mathscr{P}^{\prime}$, and $\boldsymbol{\alpha}^{\prime}$ are vector-like (thus, not director-like). The meaning of these SOS relationships is: when an electric field or a magnetic field is applied to a ferro-rotational system, the induced polarization or magnetization can have a component along the direction perpendicular to the applied $\boldsymbol{E} / \boldsymbol{H}$ and also to the $\boldsymbol{\alpha}$ direction. In other words, in ferro-rotational systems in the presence of an external electric field or magnetic field, the induced polarization or magnetization can be tilted toward the direction perpendicular to the applied $\boldsymbol{E} / \boldsymbol{H}$ and also to the $\boldsymbol{\alpha}$ direction. The tiled induced polarization can be a tilted current in ferro-rotational conductors, so a Hall-type voltage can develop without any external magnetic field. As far as we are aware of, any observations relevant to these SOS relationships have not theoretically and experimentally reported yet.

\section{Permutable SOS of $\mathscr{C} \cdot \mathscr{P}^{\prime} \approx \boldsymbol{a}^{\prime}, \boldsymbol{\alpha} \cdot \boldsymbol{\alpha}^{\prime} \approx \mathscr{D}^{\prime}$, and $\mathscr{P} \cdot \mathscr{D}^{\prime} \approx \mathscr{P}^{\prime}$}

We have also the dot product-type, rather than cross producttype, SOS relationships of $\mathscr{C} \cdot \mathscr{P}^{\prime} \approx \boldsymbol{a}^{\prime}, \boldsymbol{a} \cdot \boldsymbol{\alpha}^{\prime} \approx \mathscr{D}^{\prime}$, and $\mathscr{P} \cdot \mathscr{D}^{\prime} \approx \mathscr{P}^{\prime}$, as well as their permutable sOS relationships, summarized below: The broken symmetry operations that do not cancel on the left-hand side are colored in green as the resultant broken symmetries on the right-hand side, while canceled ones are colored in dark gray. Note that a dot product between two 1D objects means the coexistence of two 1D objects, and when a symmetry is broken in both 1D objects that are coupled to each other and act as a new 1D object, the symmetry can be not broken in the $3^{\text {rd }} 1 \mathrm{D}$ object. For this situation, we have the following SOS relationships.

\begin{tabular}{|c|c|c|c|c|}
\hline$(1) \mathcal{C} \bullet \mathscr{P}^{\prime}$ & $\approx$ Broken $\{\mathrm{I}, \mathrm{M}, \underline{\mathbf{M}}\}$ & $\otimes$ Broken $\{\mathbf{R}, \mathbf{I}, \mathbf{M}, \mathrm{T}\}$ & $=$ Broken $\{\mathbf{R}, \underline{\mathbf{M}}, \mathbf{T}\}$ & $\approx \boldsymbol{a}^{\prime}$ \\
\hline (2) $\mathcal{C}^{\prime} \cdot \mathfrak{P}$ & $\approx$ Broken $\{\mathrm{I}, \mathrm{M}, \underline{\mathrm{M}}, \mathrm{T}\}$ & $\otimes$ Broken $\{\mathbf{R}, \mathbf{I}, \mathbf{M}\}$ & $=\operatorname{Broken}\{\mathbf{R}, \underline{\mathbf{M}}, \mathbf{T}\}$ & $\approx \boldsymbol{a}^{\prime}$ \\
\hline (3) $\mathcal{C}^{\prime} \bullet \mathcal{P}^{\prime}$ & $\approx$ Broken $\{\mathrm{I}, \mathrm{M}, \underline{\mathrm{M}}, \mathrm{T}\}$ & $\otimes$ Broken $\{\mathbf{R}, \mathrm{I}, \mathbf{M}, \mathrm{T}\}$ & $=$ Broken $\{\mathbf{R}, \underline{\mathbf{M}}\}$ & $\approx a$ \\
\hline (4) $a \cdot \mathcal{e}^{\prime}$ & $\approx$ Broken $\{\mathbf{R}, \underline{M}\}$ & $\otimes$ Broken $\{\mathbf{I}, \mathbf{M}, \underline{M}, \mathbf{T}\}$ & $=$ Broken $\{\mathbf{R}, \mathbf{I}, \mathbf{M}, \mathbf{T}\}$ & $\approx \mathcal{P}^{\prime}$ \\
\hline (5) $a^{\prime} \bullet \mathfrak{e}^{\prime}$ & $\approx$ Broken $\{\mathbf{R}, \underline{\mathbf{M}}, \mathbf{T}\}$ & $\otimes$ Broken $\{\mathbf{I}, \mathbf{M}, \mathbf{M}, \mathbf{T}\}$ & $=$ Broken $\{\mathbf{R}, \mathbf{I}, \mathbf{M}\}$ & $\approx \mathscr{P}$ \\
\hline (6) $a^{\prime} \cdot c$ & $\approx$ Broken $\{\mathbf{R}, \underline{\mathbf{M}}, \mathbf{T}\}$ & $\otimes$ Broken $\{\mathbf{I}, \mathbf{M}, \underline{M}\}$ & $=$ Broken $\{\mathbf{R}, \mathbf{I}, \mathbf{M}, \mathbf{T}\}$ & $\approx \mathcal{P}^{\prime}$ \\
\hline (7) $\mathfrak{P} \bullet a^{\prime}$ & $\approx$ Broken $\{\mathbf{R}, \mathbf{I}, \mathbf{M}\}$ & $\otimes$ Broken $\{\mathrm{R}, \underline{\mathrm{M}}, \mathrm{T}\}$ & $=$ Broken $\{\mathbf{I}, \mathbf{M}, \underline{\mathbf{M}}, \mathbf{T}\}$ & $\approx \mathcal{e}^{\prime}$ \\
\hline (8) $\mathcal{P}^{\prime} \bullet a$ & $\approx$ Broken $\{\mathbb{R}, \mathbf{I}, \mathbf{M}, \mathbf{T}\}$ & $\otimes$ Broken $\{\mathbb{R}, \underline{M}\}$ & $=$ Broken $\{\mathbf{I}, \mathbf{M}, \underline{\mathbf{M}}, \mathbf{T}\}$ & $\approx \mathfrak{e}^{\prime}$ \\
\hline (9) $\mathcal{P}^{\prime} \bullet \boldsymbol{a}^{\prime}$ & $\approx$ Broken $\{\mathbb{R}, \mathbf{I}, \mathbf{M}, \mathrm{T}\}$ & $\otimes$ Broken $\{\mathrm{R}, \underline{\mathrm{M}}, \mathrm{T}\}$ & $=$ Broken $\{\mathbf{I}, \mathbf{M}, \underline{\mathbf{M}}\}$ & $\approx e$ \\
\hline (10) $\mathfrak{P} \bullet a$ & $\approx$ Broken $\{\mathrm{R}, \mathrm{I}, \mathbf{M}\}$ & $\otimes$ Broken $\{\mathrm{R}, \underline{\mathrm{M}}\}$ & $=$ Broken $\{\mathbf{I}, \mathbf{M}, \underline{\mathbf{M}}\}$ & $\approx \mathcal{e}$ \\
\hline (11) $a \bullet a^{\prime}$ & $\approx$ Broken $\{\mathbb{R}, \underline{M}\}$ & $\otimes$ Broken $\{\mathbb{R}, \underline{M}, T\}$ & $=$ Broken $\{\mathbf{T}\}$ & $\approx \mathcal{D}^{\prime}$ \\
\hline (12) $\mathfrak{P} \bullet \mathfrak{D}^{\prime}$ & $\approx$ Broken $\{\mathbf{R}, \mathbf{I}, \mathbf{M}\}$ & $\otimes$ Broken $\{\mathbf{T}\}$ & $=$ Broken $\{\mathbf{R}, \mathbf{I}, \mathbf{M}, \mathbf{T}\}$ & $\approx \mathscr{P}^{\prime}$ \\
\hline
\end{tabular}

Note that $\mathscr{C}, \mathscr{C}^{\prime}, \mathscr{D}$, and $\mathscr{D}^{\prime}$ are director-like (i.e., not-broken $\{\mathbf{R}\}$ ), so the resulting SOS orientation is fixed by vector-like objects of $\mathscr{P}$, $\boldsymbol{a}, \mathscr{P}^{\prime}$, or $\boldsymbol{a}^{\prime}$. Thus, the directions of $\mathscr{C}, \mathscr{D}^{\prime}$, and $\mathscr{C}^{\prime}$ in Fig. 5 can be in the vertical, horizontal, or out-of-plane direction. (1)-(9) list all SOS relationships permutable from $\mathscr{C} \cdot \mathscr{P}^{\prime} \approx \boldsymbol{a}^{\prime}$ : each SOS relationship has two [']s. Similar sets of permutable SOS relationships are possible from (10) to (12). First, Fig. 2c/d, reproduced in Fig. 5a, and Fig. 2h/i correspond to (2) and (12), respectively. When $\boldsymbol{E}$ and $\boldsymbol{M}$ in Fig. $2 \mathrm{c} / \mathrm{d}$ are replaced by $\boldsymbol{H}$ and $\boldsymbol{P}$, respectively, it corresponds to (5). This demonstrates a permutable SOS relationship as well as the permutable (and duality) nature of linear magnetoelectric effects. Note also that (2) and (6) mean that all diagonal linear magnetoelectrics belongs to $\mathscr{C}^{\prime}$ and any specimen constituents having SOS with $\mathscr{C}^{\prime}$ exhibit diagonal linear magnetoelectric effects. Table 1 lists the complete set of dot product-type SOS relationships among eight kinds of 1D objects (vector- or director-like quantities).

Permutable SOS relationships of (2), (5), and (7) can be also well exemplified in hexagonal rare-earth ferrites ${ }^{22}$. The $A_{2}$ phase of the system carries $\boldsymbol{P}(\mathscr{P})$ along the $c$ axis and in-plane magnetic monopole $\left(\mathscr{C}^{\prime}\right)$, and a net magnetic moment $\boldsymbol{M}\left(\boldsymbol{\alpha}^{\prime}\right)$ along the $C$ axis is, indeed, observed experimentally, which is neatly consistent with (2). Through a spin reorientation transition, the magnetic state transforms into the $\mathrm{A}_{1}$ phase with magnetic toroidal moments $\left(\mathscr{P}^{\prime}\right)$, 
(a)
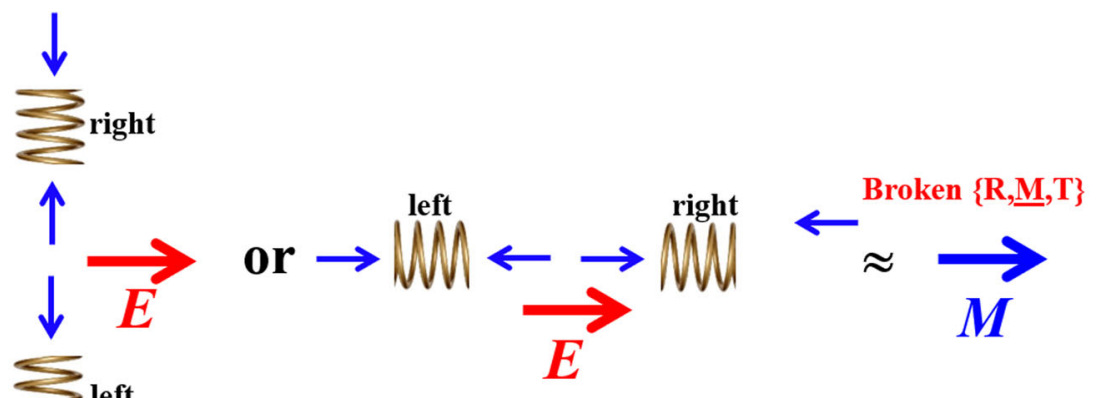

(b)

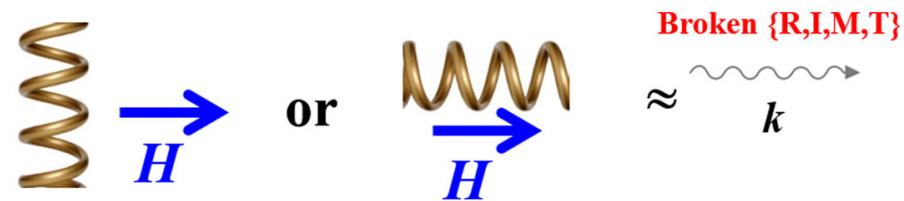

(c)
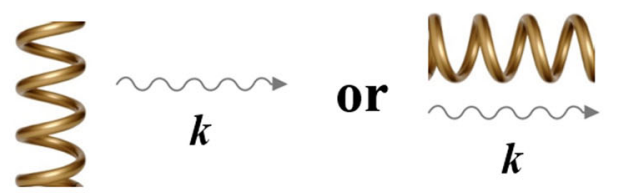

Broken $\{\mathbf{R}, \underline{\mathrm{M}}, \mathbf{T}\}$

(d)

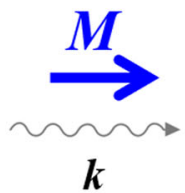

Broken $\{\mathbf{I}, \mathbf{M}, \underline{\mathbf{M}}\}$

$\approx$

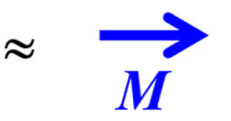

(e)
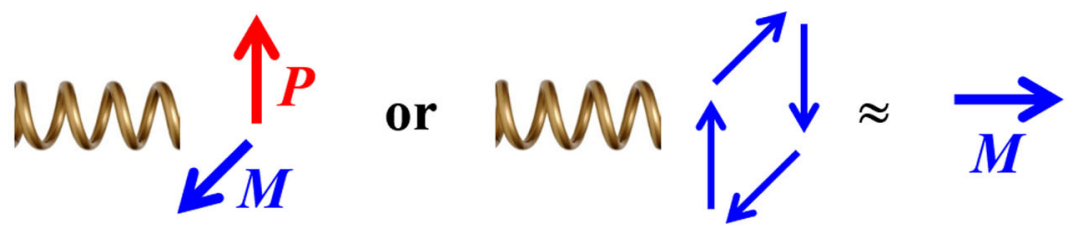

(f)

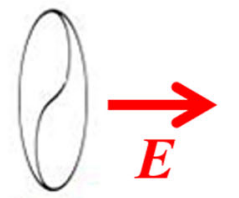

Broken $\{\mathbf{I}, \mathbf{M}, \underline{\mathrm{M}}, \mathrm{T}\}$

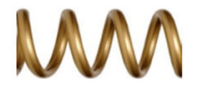

$\approx N M N$

(g)
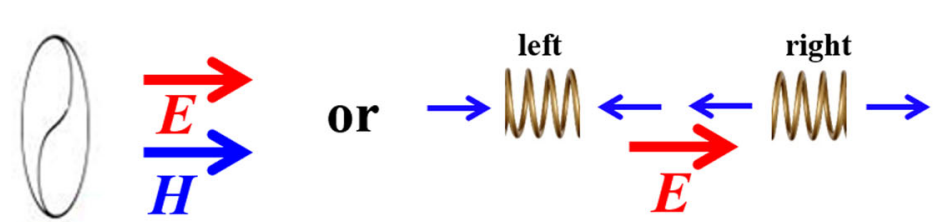

Broken $\{\mathbf{R}, \mathbf{I}, \mathbf{M}, \mathbf{T}\}$

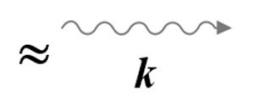

Fig. 5 Various SOS relationships. The Yin-yang symbol represents ferro-rotation. a-g correspond to $\mathscr{C}^{\prime} \cdot \mathscr{P} \approx \boldsymbol{a}^{\prime}, \boldsymbol{a}^{\prime} \cdot \mathscr{C} \approx \mathscr{P}^{\prime}, \mathscr{C} \cdot \mathscr{P}^{\prime} \approx \boldsymbol{a}^{\prime}, \mathscr{P}^{\prime}$. $\boldsymbol{a}^{\prime} \approx \mathscr{C}, \mathscr{C} \cdot \mathscr{P} \approx \boldsymbol{a}^{\prime}, \mathscr{P} \cdot \boldsymbol{\alpha} \approx \mathscr{C}$, and $\boldsymbol{a}^{\prime} \cdot \mathscr{C} \approx \mathscr{P} \cdot \mathscr{D}^{\prime} \approx \mathscr{P}^{\prime}$, respectively.

where the net magnetic moment $\boldsymbol{M}\left(\boldsymbol{\alpha}^{\prime}\right)$ is no longer allowed according to $\mathscr{P} \cdot \mathscr{P}^{\prime} \approx \mathscr{D}^{\prime}$. This is another premium example of how these permutable SOS relationships can be used to explain and predict physical properties in real materials.

Figure $5 \mathrm{~b}$ corresponds to (6), which explains longitudinal and also transverse magnetochiral effects, i.e., non-reciprocal electronic transport or optical effects in chiral materials in the presence of external magnetic fields ${ }^{23}$. Note that a twisted graphene bilayer is chiral, so exhibits a natural optical activity ${ }^{24}$, and should exhibit non-reciprocal optical and also transport effects in the presence of magnetic fields, which need to be experimentally verified. Their inverse effects in Fig. 5c, corresponding to (1), include magnetization induced by electric current in chiral materials and spinpolarized tunneling in chiral materials. The magnetization induced by electric current in chiral Te single crystals has been reported in a Faraday-type optical experiment as well as a direct magnetization 
pulse measurement ${ }^{25,26}$. The chirality-dependent spin-polarization of tunneling current was recently observed in chiral $\mathrm{Co}_{1 / 3} \mathrm{NbS}_{2}{ }^{27}$ (see below). The existence of electric current in a magnetic field along the electric current direction corresponds to (9), so it corresponds to a chiral object (see Fig. 5d). In fact, this SOS relationship is closely related to the so-called chiral anomaly in topological Weyl semimetals, which are often non-chiral crystallographically ${ }^{28-31}$. Since the toroidal moment, just like $\boldsymbol{k}$, also belongs to $\mathscr{P}^{\prime}$, the SOS in Fig. 5e should also work.

Chiral materials can exhibit natural optical activity, i.e., the rotation of light polarization of transmitted linearly-polarized light. Thus, Fig. $5 f$ SOS relationship, corresponding to (10), explains the so-called linear-gyration, i.e., the linear change of the light polarization rotation of transmitted linearly-polarized light with the varying electric field. In fact, this linear-gyration can be utilized to visualize ferro-rotational domains, since opposite ferro-rotational domains show the opposite linear-gyration effects ${ }^{1}$. This idea was, indeed, confirmed in the visualization of ferro-rotational domains in ilmenite $\mathrm{NiTiO}_{3}$, as shown in Fig. $6 b^{32}$. There exists a large number of ferro-rotational compounds with point group symmetry of $\overline{1}, 2 / m, \overline{3}, \overline{4}, 4 / m, \overline{6}$ or $6 / m^{33}$. Examples include $\mathrm{Cu}_{3} \mathrm{Nb}_{2} \mathrm{O}_{8}(P \overline{1})^{33}$,

Table 1. Dot product-type SOS relationships among eight kinds of 1D objects (vector- or director-like quantities).

\begin{tabular}{l|cccccccc}
\hline \multicolumn{2}{c}{$\mathscr{D}^{2}$} & $\mathscr{C}$ & $\mathscr{P}$ & $\boldsymbol{a}$ & $\mathscr{D}^{\prime}$ & $\mathscr{C}^{\prime}$ & $\mathscr{P}^{\prime}$ & $\boldsymbol{a}^{\prime}$ \\
\hline $\mathscr{D}$ & $\mathscr{D}$ & $\mathscr{C}$ & $\mathscr{P}$ & $\boldsymbol{a}$ & $\mathscr{D}^{\prime}$ & $\mathscr{C}^{\prime}$ & $\mathscr{P}^{\prime}$ & $\boldsymbol{a}^{\prime}$ \\
$\mathscr{C}$ & $\mathscr{C}$ & $\mathscr{D}$ & $\boldsymbol{a}$ & $\mathscr{P}$ & $\mathscr{C}^{\prime}$ & $\mathscr{D}^{\prime}$ & $\boldsymbol{a}^{\prime}$ & $\mathscr{P}^{\prime}$ \\
$\mathscr{P}$ & $\mathscr{P}$ & $\boldsymbol{a}$ & $\mathscr{D}$ & $\mathscr{C}$ & $\mathscr{P}^{\prime}$ & $\boldsymbol{a}^{\prime}$ & $\mathscr{D}^{\prime}$ & $\mathscr{C}^{\prime}$ \\
$\boldsymbol{a}$ & $\boldsymbol{a}$ & $\mathscr{P}$ & $\mathscr{C}$ & $\mathscr{D}$ & $\boldsymbol{a}^{\prime}$ & $\mathscr{P}^{\prime}$ & $\mathscr{C}^{\prime}$ & $\mathscr{D}^{\prime}$ \\
$\mathscr{D}^{\prime}$ & $\mathscr{D}^{\prime}$ & $\mathscr{C}^{\prime}$ & $\mathscr{P}^{\prime}$ & $\boldsymbol{a}^{\prime}$ & $\mathscr{D}$ & $\mathscr{C}$ & $\mathscr{P}$ & $\boldsymbol{a}$ \\
$\mathscr{C}^{\prime}$ & $\mathscr{C}^{\prime}$ & $\mathscr{D}^{\prime}$ & $\boldsymbol{a}^{\prime}$ & $\mathscr{P}^{\prime}$ & $\mathscr{C}$ & $\mathscr{D}$ & $\boldsymbol{a}$ & $\mathscr{P}$ \\
$\mathscr{P}^{\prime}$ & $\mathscr{P}^{\prime}$ & $\boldsymbol{a}^{\prime}$ & $\mathscr{D}^{\prime}$ & $\mathscr{C}^{\prime}$ & $\mathscr{P}$ & $\boldsymbol{a}$ & $\mathscr{D}$ & $\mathscr{C}$ \\
$\boldsymbol{a}^{\prime}$ & $\boldsymbol{C}^{\prime}$ & $\mathscr{P}^{\prime}$ & $\mathscr{C}^{\prime}$ & $\mathscr{D}^{\prime}$ & $\boldsymbol{a}$ & $\mathscr{P}$ & $\mathscr{C}$ & $\mathscr{D}$ \\
\hline
\end{tabular}

$\mathrm{RbFe}\left(\mathrm{MoO}_{4}\right)_{2}(P \overline{3} \text { below } 190 \mathrm{~K})^{34}, \mathrm{CaMn}_{7} \mathrm{O}_{12}(R \overline{3} \text { below } 440 \mathrm{~K})^{35}$, $\mathrm{NbO}_{2}\left(14_{1} / a \text { below } 1081 \mathrm{~K}\right)^{36}$, and $\mathrm{Pb}_{2} \mathrm{CoTeO}_{6}(R \overline{3} \text { below } 370 \mathrm{~K})^{37^{\prime}}$. Ferro-rotational domain visualization of these compounds needs to be further investigated with the linear-gyration technique.

Figure $6 a$ exhibits the atomic model of a topological vortex surrounded by six structural chiral domain boundaries in $\mathrm{Co}_{1 / 3} \mathrm{NbS}_{2}$. Since Co intercalants occupy one of three possible $\mathrm{Nb}$-atop sites, A, B, and $C$, there exist six possible combinations considering the two layers within a unit cell, exhibiting A/B-type stacking. Solid and dashed lines refer to structural chiral domain boundaries in the first and the second layer, respectively. As a result of intercalation site change, the chiral distortion of the $S$ layer reverses as depicted by red and blue triangular arrows and gives the alternating structural chirality across the vortex domain boundaries. In reference to the SOS relationship shown in Fig. 5c, flowing electric current across the chiral structure induces a magnetization along the current direction, which should work even for local probes such as scanning tunneling microscopy (STM). Figure 6b shows the spin-polarized STM (SP-STM) measurement around a topological vortex that clearly reveals the alternating domain contrast locked to the chirality of each domain ${ }^{27}$. It is noteworthy that the induced domain contrast does not require long-range ordered magnetic moments, in contrast to the conventional SP-STM. Instead, the specimen constituent comprised of the chiral structure and the tunneling current gives the same set of broken symmetries as magnetization, as shown in Fig. 5c.

We would like to note finally that ferro-rotation is rather common in numerous materials ${ }^{33-37}$, but does not break inversion symmetry, so difficult to be coupled with measuring probes and thus has been studied only to a limited degree. The traditional way to study ferrorotational domains is atomic-resolved transmission electron microscopy (TEM) such as high-resolution TEM or high-angle annular darkfield (HAADF)-STEM to observe the ferro-rotational distortions or ferro-rotational domains ${ }^{32}$. Figure 6 e depicts a pair of ferro-rotational domains, corresponding to a clockwise and counterclockwise rotation of the $\mathrm{FeO}_{6}$ octahedra in $\mathrm{RbFe}\left(\mathrm{MoO}_{4}\right)_{2}$. Utilizing the cryogenic dark-field TEM technique (Fig. $6 \mathrm{f}$ ), the contrast difference between the two ferro-rotational domains shows up as the brighter (a) $\mathrm{Co}_{1 / 3} \mathrm{NbS}_{2}$
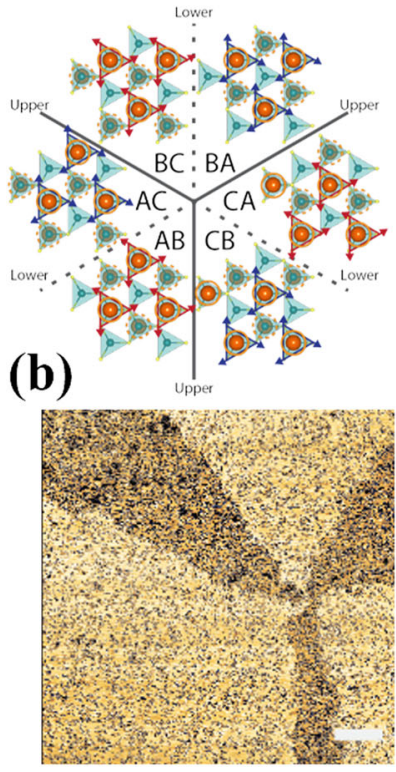

(c)

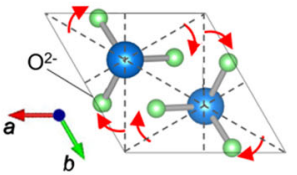

A+ domain
$\mathrm{NiTiO}_{3}$

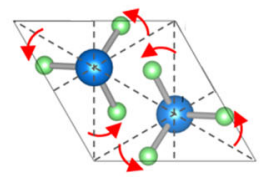

A-domain $\theta=45^{\circ}$

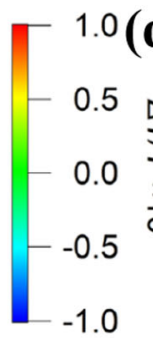

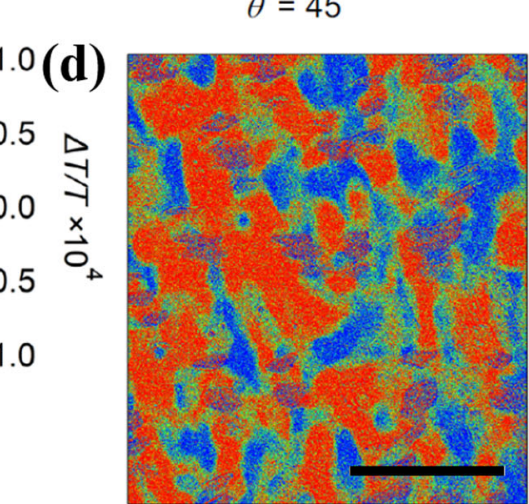

(e) $\mathrm{RbFeMo}_{2} \mathrm{O}_{8}$
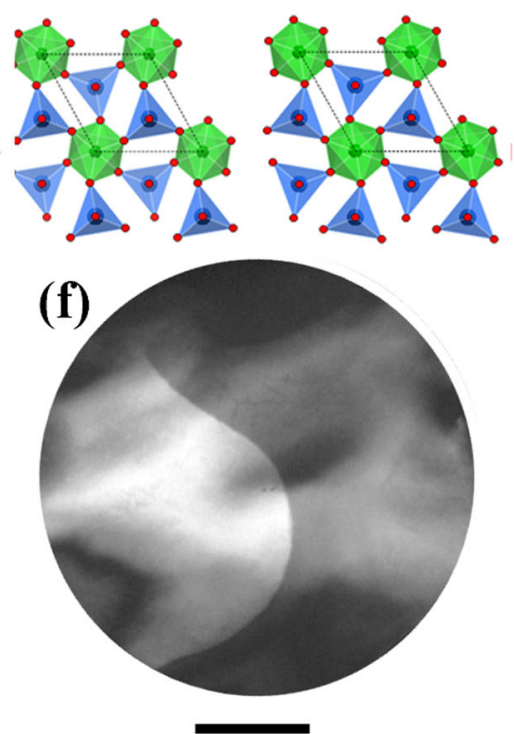

Fig. 6 Visualization of domains associated with various broken symmetries. a A $Z_{6}$ vortex of $\mathrm{Co}_{1 / 3} \mathrm{NbS}_{2}$ with chiral domains. $\mathbf{b} \mathrm{Chirality}^{-}$ dependent SP-STM unveils a $Z_{6}$ vortex ${ }^{27}$. (scale bar $=20 \mathrm{~nm}$ ). c The atomic structure of ferro-rotational domains of $\mathrm{NiTiO}_{3}{ }^{32}$. d Ferro-rotational domains in $\mathrm{NiTiO}_{3}$ visualized with an electro-gyration technique. (scale bar $=100 \mu \mathrm{m}$ ). e The atomic structure of ferro-rotational domains of $\mathrm{RbFe}\left(\mathrm{MoO}_{4}\right)_{2}$. f Ferro-rotational domains observed in TEM on $\mathrm{RbFe}\left(\mathrm{MoO}_{4}\right)_{2}^{38}($ scale bar $=500 \mathrm{~nm})$. 
$G_{a}$ spot for the left domain and the darker $G_{b}$ spot for the right domain are collected. Note that the Bragg spot $G_{a}$ is converted into $G_{b}$ in the reciprocal space by the twofold rotation as two ferrorotation domains do in real space ${ }^{38}$. The SOS relations of ferrorotation such as Fig. $5 \mathrm{f}, \mathrm{g}$ can be good guidance for the further exploration of ferro-rotations in the future.

In summary, the recent exotic experimental results such as electric current-induced magnetization in strained monolayer $\mathrm{MoS}_{2}$, NLHE in monolayer WTe ${ }_{2}$, spin-polarized tunneling in chiral $\mathrm{Co}_{1 / 3} \mathrm{NbS}_{2}$ in a paramagnetic state, and non-reciprocal electronic transport in polar BiTeBr can be understood in terms of permutable SOS relationships of 1D objects. We have presented all possible permutable SOS relationships among eight kinds of 1D objects. Based on the permutable SOS relationship, we have proposed a large number of new phenomena, which can be experimentally verified in the future. New predictions include (but are not limited to): (1) a twisted graphene bilayer in the presence of magnetic fields should exhibit non-reciprocal transport and optical effects Note 139 , (2) NLHE in strained monolayer $\mathrm{MoS}_{2}$, (3) electric current-induced magnetization in monolayer $\mathrm{Mo}(\mathrm{W}) \mathrm{Te}_{2}$, (4) electric current-induced magnetization, NLHE, and non-reciprocal electronic transport in bulk polar conductors such as $\mathrm{Ca}_{3} \mathrm{Ru}_{2} \mathrm{O}_{7}, \mathrm{GeTe}, \mathrm{TaAs}$, and $\mathrm{BiTel}(\mathrm{Br}$, $\mathrm{Cl})$, (5) light- or thermal current-induced magnetization in numerous polar materials, (6) electric/thermal current-, or light-induced magnetization of polar materials at polar domain walls, (7) CDHE in any materials with enough electric conduction, (8) Ettingshausen, Nernst, or thermal Hall effect versions of CDHE in any materials with circularly-polarized light (or vortex beam with orbital angular momentum) illumination without any applied magnetic field, (9) non-reciprocal spin wave propagation in polar materials such as $\mathrm{Fe}$ $(\mathrm{Mn}, \mathrm{Ni}, \mathrm{Zn})_{2} \mathrm{Mo}_{3} \mathrm{O}_{8}$ or $\mathrm{Ni}_{3} \mathrm{TeO}_{6}$ in magnetic fields, (10) non-reciprocal directional dichroism in $\mathrm{a}-\mathrm{Fe}_{2} \mathrm{O}_{3}$ below the Morin transition in the presence of an electric field, (11) visualization of ferro-rotational domains of ferro-rotational compounds such as $\mathrm{RbFe}\left(\mathrm{MoO}_{4}\right)_{2}$, $\mathrm{CaMn}_{7} \mathrm{O}_{12}, \mathrm{Cu}_{3} \mathrm{Nb}_{2} \mathrm{O}_{8}, \mathrm{NbO}_{2}$, and $\mathrm{Pb}_{2} \mathrm{CoTeO}_{6}$ using a lineargyration technique, (12) non-reciprocal directional dichroism of electric current, spin wave or light in ferro-rotational systems in the presence of both electric and magnetic fields, and (13) the tiled induced polarization (current) and magnetization in ferro-rotational systems in the presence of an external electric field and magnetic field, respectively. We also find this important systematics: all magnetic states having SOS with $\mathscr{C}^{\prime}$ exhibit diagonal linear magnetoelectric effects, and all magnetic states having SOS with $\mathscr{P}^{\prime}$ exhibit off-diagonal linear magnetoelectric effects. It will be exuberating to confirm these predictions in bulk quantum materials as well as $2 \mathrm{D}$ materials in the coming years. The following poem summaries our findings in an esthetic manner:

Symmetry defines evident beauty.

Once broken, it brings observables.

Unveiling duality and permutability.

These tricks that Nature plays become the crystal ball to see its hidden beauty.

In Nature, nothing is perfect, yet everything is perfect. Note 2

Note 1: After submission of our manuscript, the following paper $^{39}$, directly relevant to this prediction, has published.

Note 2: Walker A. In nature, nothing is perfect and everything is perfect. Trees can be contorted, bent in weird ways, and they're still beautiful.

\section{METHODS}

\section{SOS analysis}

The relationships between quasi-1D specimen constituents (lattice distortions or spin arrangements in external magnetic field, electric fields or stress, and also their time evolution) and measuring probes/quantities (observables such as transmitting electrons, phonons, magnons, photons in various polarization states, including electrons or photons with spin or orbital angular momentum, bulk magnetization or polarization, and also experimental setups to measure, e.g., Hall-type effects) are examined in terms of their characteristics under various symmetry operations of rotation, space inversion, mirror reflection, and time reversal. When quasi1D specimen constituents have identical or more (but not less) broken symmetries, compared with measuring probes/quantities, except translation symmetry and rotation symmetry around the 1D direction, they are said to exhibit SOS, and the corresponding phenomena can take place. In terms of SOS, we have considered the specimen constituents for light- or current-induced magnetization, NLHE, the spin polarization of electric current in non-magnetic or paramagnetic states, linear magneto-electric effects, and natural optical activity or non-reciprocal effects in the presence of an electric field. In addition, we have also discussed what new phenomena can be observed in ferro-rotational systems from SOS considerations.

\section{DATA AVAILABILITY}

The datasets generated during the current study are available from the corresponding author on reasonable request.

Received: 2 January 2021; Accepted: 7 April 2021; Published online: 11 June 2021

\section{REFERENCES}

1. Cheong, S.-W. SOS: symmetry operational similarity. npj Quant. Mater. 4, 53 (2019).

2. Cheong, S.-W. Trompe L'oeil ferromagnetism. npj Quant. Mater. 5, 37 (2020).

3. Cheong, S.-W., Talbayev, D., Kiryukhin, V. \& Saxena, A. Broken symmetries, nonreciprocity, and multiferroicity. npj Quant. Mater. 3, 19 (2018).

4. Hlinka, J. Eight types of symmetrically distinct vectorlike physical quantities. Phys. Rev. Lett. 113, 165502 (2014).

5. Popov, Y. F., Kadomtseva, A. M., Belov, D. V. \& Vorob'ev, G. P. Magnetic-fieldinduced toroidal moment in the magnetoelectric $\mathrm{Cr}_{2} \mathrm{O}_{3}$. JETP Lett. 69, 330-335 (1999).

6. Krichevtsov, B. B., Pavlov, V. V., Pisarev, R. V. \& Gridnev, V. N. Spontaneous nonreciprocal reflection of light from antiferromagnetic $\mathrm{Cr}_{2} \mathrm{O}_{3}$. J. Phys. Condens. Matter 5, 8233-8244 (1993).

7. $\mathrm{Yu}, \mathrm{S}$. et al. High-temperature terahertz optical diode effect without magnetic order in polar FeZnMo $\mathrm{O}_{3}$. Phys. Rev. Lett. 120, 037601 (2018).

8. Ideue, T. et al. Bulk rectification effect in a polar semiconductor. Nat. Phys. 13, 578-583 (2017).

9. Yokosuk, M. et al. Nonreciprocal directional dichroism of a chiral magnet in the visible range. npj Quant. Mater. 5, 20 (2020).

10. $\mathrm{Ma}, \mathrm{Q}$. et al. Observation of the nonlinear Hall effect under time-reversalsymmetric conditions. Nature 565, 337-342 (2019).

11. Lee, J., Wang, Z., Xie, H., Mak, K. F. \& Shan, J. Valley magnetoelectricity in singlelayer $\mathrm{MoS}_{2}$. Nat. Mat. 16, 887-891 (2017).

12. Son, J., Kim, K.-H., Ahn, Y. H., Lee, H.-W. \& Lee, J. Strain engineering of the Berry curvature dipole and valley magnetization in monolayer $\mathrm{MoS}_{2}$. Phys. Rev. Lett. 123, 036806 (2019).

13. Yoshida, Y. et al. Crystal and magnetic structure of $\mathrm{Ca}_{3} \mathrm{Ru}_{2} \mathrm{O}_{7}$. Phys. Rev. B 72, 054412 (2005).

14. Rabe, K. M. \& Joannopoulos, J. D. Theory of the structural phase transition of GeTe. Phys. Rev. B 36, 6631 (1987).

15. Liang, W. et al. Giant anisotropic nonlinear optical response in transition metal monopnictide Weyl semimetals. Nat. Phys. 13, 350-355 (2017).

16. Ishizaka, K. et al. Giant Rashba-type spin splitting in bulk BiTel. Nat. Mater. 10, 521-526 (2011).

17. Qiong, M. et al. Observation of the nonlinear Hall effect under time-reversalsymmetric conditions. Nat. Mater. 565, 337-342 (2019).

18. Zhou, Y. \& Liu, F. Realization of an antiferromagnetic superatomic graphene: Dirac Mott insulator and circular dichroism Hall effect. Nano Lett. 21, 230-235 (2021).

19. Ivchenko, E. L. \& Pikus, G. E. New photogalvanic effect in gyrotropic crystals (Pis'ma Zh. Eksp. Teor. Fiz. 27, 640 (1978)). JETP Lett. 27, 604-608 (1978).

20. Belinicher, V. I. Space-oscillating photocurrent in crystals without symmetry center. Phys. Lett. A 66, 213-214 (1978).

21. Asnin, V. M. et al. Observation of a photo-emf that depend on the sign of the circular polarization of the light. (Pis'ma Zh. Eksp. Teor. Fiz. 28, 80-84 (1978)). JETP Lett. 28, 74-77 (1978). 
22. Du, K. et al. Vortex ferroelectric domains, large-loop weak ferromagnetic domains, and their decoupling in hexagonal $(\mathrm{Lu}, \mathrm{Sc}) \mathrm{FeO}_{3}$. npj Quant. Mater. 3, 33 (2018).

23. Nakagawa, N. et al. Magneto-chiral dichroism of $\mathrm{CsCuCl}_{3}$. Phys. Rev. B 96, 121102 (R) (2017).

24. Kim, C. J. et al. Chiral atomically thin films. Nat. Nanotechnol. 11, 520-524 (2016).

25. Vorobev, L. E. et al. Optical-activity in tellurium induced by a current. JETP Lett. 29, 441-445 (1979).

26. Furukawa, T., Shimokawa, Y., Kobayashi, K. \& Itou, T. Observation of current induced bulk magnetization in elemental tellurium. Nat. Commun. 8, 954 (2017).

27. Lim, S. J., Huang, F.-T., Pan, S., Wang, K. F., Kim, J.-W. \& Cheong, S.-W. Magnetochiral spin-polarized tunneling in a paramagnetic state, Preprint at https:// www.researchsquare.com/article/rs-70373/v1.

28. Zyuzin, A. A., Wu, S. \& Burkov, A. A. Weyl semimetal with broken time reversal and inversion symmetries. Phys. Rev. B 85, 165110 (2012).

29. Burkov, A. A. \& Balents, L. Weyl semimetal in a topological insulator multilayer. Phys. Rev. Lett. 107, 127205 (2011).

30. Bell, J. S. \& Jackiw, R. A PCAC puzzle: $\pi 0 \rightarrow \gamma \gamma$ in the $\sigma$-model. Nuovo Cim. A $6 \mathbf{6 0}$ 47-61 (1969).

31. Adler, S. L. Axial-vector vertex in spinor electrodynamics. Phys. Rev. 177, 2426-2438 (1969).

32. Hayashida, T. et al. Visualization of ferroaxial domains in an order-disorder type ferroaxial crystal. Nat. Commun. 11, 4582 (2020).

33. Johnson, R. D. et al. $\mathrm{Cu}_{3} \mathrm{Nb}_{2} \mathrm{O}_{8}$ : a multiferroic with chiral coupling to the crystal structure. Phys. Rev. Lett. 107, 137205 (2011).

34. Jin, W. et al. Observation of a ferro-rotational order coupled with second-order nonlinear optical fields. Nat. Phys. 16, 42-46 (2020).

35. Johnson, R. D. et al. Giant improper ferroelectricity in the ferroaxial magnet $\mathrm{CaMn}_{7} \mathrm{O}_{12}$. Phys. Rev. Lett. 108, 067201 (2012).

36. Pynn, R., Axe, J. D. \& Thomas, R. Structural distortions in the low-temperature phase of $\mathrm{NbO}_{2}$. Phys. Rev. B 13, 2965-2975 (1976).

37. Ivanov, S. A., Nordblad, P., Mathieu, R., Tellgren, R. \& Ritterd, C. Structural and magnetic properties of the ordered perovskite $\mathrm{Pb}_{2} \mathrm{CoTeO}_{6}$. Dalton Trans. 39, 11136-11148 (2010).

38. Huang, F.-T., Admasu, A. S., Han, M. G. \& Cheong, S.-W. TEM on ferro-rotational domains in $\mathrm{RbFeMo}_{2} \mathrm{O}_{8}$. To be published.

39. Liu, Y., Holder, T. \& Yan, B. Chirality-induced giant unidirectional magnetoresistance in twisted bilayer graphene. Innovation 2, 100085 (2021).

\section{ACKNOWLEDGEMENTS}

This work was supported by the DOE under Grant no. DOE: DE-FG02-07ER46382 and by the Gordon and Betty Moore Foundation's EPiQS Initiative through Grant
GBMF4413 to the Rutgers Center for Emergent Materials. We have greatly benefited from discussions with Sobhit Singh, David Vanderbilt, Daniel Khomskii, and Venkat Goplan.

\section{AUTHOR CONTRIBUTIONS}

S.W.C. conceived and supervised the project. All authors were involved in various symmetry analyses. S.L. and F.-T.H. wrote the STM and TEM parts, respectively, K.D. wrote some of the example parts and S.W.C. wrote the remaining part.

\section{COMPETING INTERESTS}

The authors declare no competing interests.

\section{ADDITIONAL INFORMATION}

Correspondence and requests for materials should be addressed to S.-W.C.

Reprints and permission information is available at http://www.nature.com/ reprints

Publisher's note Springer Nature remains neutral with regard to jurisdictional claims in published maps and institutional affiliations.

(i) Open Access This article is licensed under a Creative Commons Attribution 4.0 International License, which permits use, sharing, adaptation, distribution and reproduction in any medium or format, as long as you give appropriate credit to the original author(s) and the source, provide a link to the Creative Commons license, and indicate if changes were made. The images or other third party material in this article are included in the article's Creative Commons license, unless indicated otherwise in a credit line to the material. If material is not included in the article's Creative Commons license and your intended use is not permitted by statutory regulation or exceeds the permitted use, you will need to obtain permission directly from the copyright holder. To view a copy of this license, visit http://creativecommons. org/licenses/by/4.0/.

(c) The Author(s) 2021 